\title{
Estrategias de las tabaqueras para captar fumadores jóvenes en España: una revisión de los documentos internos
}

\section{Tobacco industry strategies to attract young smokers in Spain: a review of companies' internal documents}

\author{
FRANCISCO Soto Mas*; Holly E. JaCOBSON ${ }^{\star *}$;

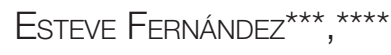

\author{
* Translational Hispanic Health Research Initiative, College of Education, \\ University of Texas at El Paso, USA \\ ${ }^{* *}$ Department of Linguistics, University of New Mexico, USA \\ ***Unidad de Control del Tabaquismo, Institut Català d'Oncologia-IDIBELL, \\ L'Hospitalet (Barcelona), España \\ ${ }^{* \star \star \star D e p a r t a m e n t o ~ d e ~ C i e n c i a s ~ C l i ́ n i c a s, ~ F a c u l t a t ~ d e ~ M e d i c i n a, ~ C a m p u s ~ d e ~}$ \\ Bellvitge, Univesitat de Barcelona, España. \\ Enviar correspondencia a: \\ Francisco Soto Mas. \\ College of Education EDUC 601. \\ University of Texas at El Paso \\ 500 W. University Ave. El Paso, Texas 79968. USA \\ E-mail: fsoto3@utep.edu \\ Teléfono: 915-747-5915. Fax: 915-747-7441
}

\section{RESUMEN}

Fundamento: Las estrategias de las compañias tabaqueras para promocionar el tabaquismo y bloquear las políticas de control son un factor clave en el consumo de tabaco entre jóvenes. La documentos internos pueden ayudar a identificar estas estrategias, conseguir apoyo social para las políticas de control y planificar actuaciones de salud pública en España. Este estudio identificó y analizó documentos relacionados con las actividades que las tabaqueras realizaron durante los años 80 y 90 para mantener y aumentar el tabaquismo entre los jóvenes españoles.

Métodos: Búsqueda electrónica por palabra clave de documentos internos de las compañias tabaqueras en la base de datos digital Legacy Tobacco Documents Library.

Resultados: Se identificaron y revisaron más de 1.000 documentos internos, como cartas, mensajes, memorandos y otros documentos. En este análisis se incluyen 17 documentos directamente relacionados con el propósito del estudio.

Conclusiones: Las tabaqueras financiaron y ejecutaron actividades de investigación, diseño, propaganda y manipulación política y social con el objetivo de mantener una alta incidencia y prevalencia de fumadores jóvenes en España. Estas actividades pueden haber influido en las actuales cifras de tabaquismo.

Palabras clave: tabaco, jóvenes, España, tabaqueras, documentos internos

\section{ABSTRACT}

Background: Tobacco companies' strategies to promote tobacco use and undermine tobacco control policy are key in tobacco use among young people. The analysis of tobacco companies' internal documents can be instrumental in identifying those strategies, improving social support for tobacco control policy, and planning public health interventions. The goal of this study was to identify and analyze internal documents related to the activities of tobacco companies during the 1980s and 90s aimed at promoting smoking in young Spaniards.

Methods: Electronic search by key word of tobacco industry documents held at the Legacy Tobacco Documents Library.

Results: More than 1,000 documents were identified and reviewed, including letters, messages, memos, and other documents. Seventeen documents related to the objective of the study are analyzed and discussed.

Conclusions: Tobacco companies funded and conducted activities aimed at maintaining high smoking incidence and prevalence in Spain. These activities, which included research, cigarette and packaging design, promotion, and social and political manipulation, may have had an impact on current levels of tobacco use in Spain.

Key words: tobacco, young people, Spain, tobacco companies, internal documents 


\section{INTRODUCCIÓN}

$\mathrm{E}$ tabaquismo en niños y adolescentes varía considerablemente entre países y regiones y es difícil establecer una prevalencia a nivel mundial porque en muchos países no se recogen datos de forma sistemática, y entre los que sí no todos utilizan las mismas variables (como consumo alguna vez en la vida, en los últimos 30 días, etc.). Pero sí se sabe que el consumo de tabaco en menores de 18 años es alarmante y en las próximas décadas el 30\% de los jóvenes se convertirán en fumadores, lo que supondrá una mortalidad anual de 10 millones de personas ${ }^{1}$. En España la perspectiva no es muy diferente. El tabaco es la droga que los escolares españoles empiezan a consumir a edad más temprana².

Independientemente de las cifras, es interesante explorar el porqué en ciertos grupos de edad, como adolescentes y adultos jóvenes, no se consigue un evidente progreso. La respuesta a esta pregunta no es tan complicada ya que se ha establecido que la influencia de las tabaqueras es determinante en la incidencia y prevalencia de tabaquismo, sobre todo en ciertos grupos de edad. Aunque la adopción del tabaquismo depende de factores genéticos, psicosociales, cognitivos, farmacológicos, psiquiátricos, políticos y contextuales ${ }^{3-5}$, y en España son muchos los "actores" que intervienen en la promoción del tabaquismo ${ }^{6}$, hay que insistir en que el comportamiento de las compañias y sus estrategias para promocionar el uso de tabaco y bloquear las políticas del control son clave en el consumo de tabaco entre menores y jóvenes. Por un lado, la influencia de la propaganda y los esfuerzos de las tabaqueras son más determinantes en los jóvenes que en los adultos, y la alta prevalencia de fumadores jóvenes en todo el mundo se puede atribuir a la constante inversión en promoción y a la persistente labor de entorpecimiento de las políticas de control por parte de las multinacionales del tabaco 7 .

Al igual que en el resto del mundo, varios estudios en nuestro medio han desvelado la influencia de las tabaqueras en los fumadores jóvenes españoles. Durante la mayor parte del siglo XX el aumento del consumo de tabaco en España estuvo directamente relacionado con las prácticas de mercado de la industria tabacalera tanto nacional como multinacional ${ }^{8}$. Igualmente, la publicidad tiene una influencia muy importante y las tabaqueras han dirigido también en España la propaganda en medios de comunicación escritos a los jóvenes $^{9-14}$. Este interés en el mercado joven español es la respuesta de las compañías al descenso en la prevalencia de consumo de tabaco que se ha venido observando entre los hombres desde los años $80^{15}$ y en la población general de 35 a 64 años desde el año 2003². Esta mejora no gusta a las compañías tabaqueras, ya que las obliga a reclutar unos 480 nuevos fumadores diarios si quieren mantener su ritmo de negocio en España ${ }^{8}$.

El propósito de este estudio fue identificar documentos internos relacionados con el interés de las tabaqueras en el mercado joven español. Aunque un reciente trabajo de revisión desveló los esfuerzos históricos de las grandes tabaqueras para estimular el uso de tabaco entre los menores y jóvenes a nivel internacionall ${ }^{16}$, el presente estudio se centra en documentos relacionados con España.

\section{MATERIAL Y MÉTODOS}

La metodología que hemos utilizado para la investigación electrónica de los documentos internos ha sido descrita en publicaciones anteriores ${ }^{16-18}$. Para este estudio la búsqueda se realizó en la biblioteca digital Legacy Tobacco Documents Library (University of California, San Francisco, USA). La base de datos contiene aproximadamente 11 millones de documentos (más de 60 millones de páginas) fechados entre 1950 y 2002 procedentes de las propias multinacionales del tabaco y otras colecciones, y permite tres niveles de búsqueda: básica, avanzada y experto. Se empleó principalmente la opción "avanzada", que permite la búsqueda mediante la combinación de hasta 6 palabras o frases (simple Boolean query) en todo el documento o en diferentes campos o secciones (como título, tipo de documento, etc.) y en una o más de las 16 colecciones diferentes que contiene la base de datos (como Philip Morris, Research, Multimedia Collection, etc.). Las palabras clave en inglés y en español, las combinaciones que se utilizaron y los resultados de la distintas búsquedas se incluyen en la Tabla 1. En todas la búsquedas se seleccionaron todas las colecciones. Dado el elevado número total de documentos que resultaron de las diferentes búsquedas, 14.383 , sólo se procedió a examinar los 1.006 documentos resultantes de la búsqueda Spain (Entire Record) and YAS (Young Adult Smoker) (Entire Record). En primer lugar se examinaron los campos de referencia (ver Tabla 2) de cada documento para determinar su relevancia de acuerdo al propósito del estudio, particularmente país (España, Spain), título (relacionado con el tema de interés para el estudio), fecha

Tabla 1. Resultado de búsqueda avanzada por palabra(s) clave. Todas las colecciones (Legacy Tobacco Documents Library. Universidad de California, San Francisco)

\begin{tabular}{l|c}
\hline Palabra clave (Campo de referencia) & No. de documentos \\
\hline Spain (Title) & 2.516 \\
España (Title) & 421 \\
Spain (Title) and Minors (Entire Record) & 27 \\
Spain (Title) and Youth (Entire Record) & 84 \\
Youth (Title) and Spain (Entire Record) & 1.006 \\
Spain (Entire Record) and YAS (Entire Record) & 10 \\
Spain (Title) and YAS (Entire Record) & 243 \\
YAS (Title) and Spain (Entire Record) & 10.109 \\
Young Adult Smoker (Entire Record) and Spain (Entire Record) & 65 \\
\hline Young Adult Smoker (Entire Record) and Spain (Title) & 14.383 \\
\hline TOTAL & 2 \\
\hline
\end{tabular}


(desde 1980) y tipo de documento (memo/memorandum, letter, note, report). Este sistema permite la preselección de documentos de una forma relativamente rápida sin tener que acceder al documento completo en PDF, imagen TIFF o página por página. Aproximadamente el $50 \%$ de los documentos fueron eliminados, y el resto visualizados y analizados. Hay que señalar que el número exacto de documentos que se seleccionaron y visualizaron es difícil de estimar, ya que en las pantallas de resultados muchos documentos aparecen repetidos porque un mismo documento se encuentra en más de una colección y porque muchos documentos se archivaron en más de un formato o con diferentes títulos o palabras clave. Además, con frecuencia las búsquedas se ampliaron para visualizar documentos relacionados que no necesariamente aparecieron en las pantallas de resultados iniciales.

\section{RESULTADOS Y DISCUSIÓN}

De entre todos los documentos visualizados y analizados para este estudio, 17 se discuten a continuación (ver Tabla 3). En la selección de estos documentos se utilizaron varios criterios, como la fecha, para facilitar la organización y discusión de los resultados de forma cronológica dentro del rango de fecha del estudio; autor, para incluir documentos tanto de tabaqueras multinacionales como españolas ya que en el proceso de preselección se evidenció la colaboración entre distintas compañias; contenido, aquéllos documentos que mejor ilustran las actividades relacionadas con el propósito del estudio; y relevancia, tipo e importancia de la información en el documento. También se incluyó un documento no fechado, por su contenido y relevancia.

Los documentos que se describen a continuación muestran el interés de las compañías en promocionar sus productos entre los menores y sus actividades a nivel político para facilitar este objetivo, además de su inversión en diseño. Los ingredientes del cigarrillo juegan un papel decisivo en la iniciación al tabaquismo y, por tanto, en su incidencia y prevalencia entre los menores y adultos jóvenes ${ }^{19}$. Igualmente, se discute la influencia de las multinacionales del tabaco en la compañias españolas y que éstas no eran ajenas a las tácti-

Tabla 2. Ejemplo de pantalla de resultados, campos de referencia (Legacy Tobacco Documents Library. Universidad de California, San Francisco)

\begin{tabular}{l|r}
\hline Title & Spain \\
\hline Date & report \\
Type & 321528762 \\
Bates & Spain \\
Country & British American Tobacco \\
Collection & 1 \\
Pages & bcmn0000 \\
File\# & 0391 \\
Box & mum63a99 \\
Added UCSF & 20050809 (August 9, 2005) \\
TID & mum7) \\
\hline
\end{tabular}

cas de las multinacionales, al contrario siempre se prestaron a colaborar con ellas y a adoptar sus estrategias.

Desde los años 60 las tabaqueras sabian que la clave para el futuro de su negocio era el reclutamiento de menores y jóvenes. En los años 80 y 90 se llevaron a cabo campañas de propaganda y captación del fumador adulto joven (YAS) como respuesta al descenso de fumadores más mayores que compañías como RJ Reynolds ya anticipaban ${ }^{20}$, y en previsión a las medidas de control a nivel internacional.

En España, Tabacalera y otras compañías más pequeñas colaboraron con las grandes multinacionales en los programas YAS. En una carta de 1986 un directivo en España de la compañía RJ Reynolds describe el éxito de la campaña Winston Americana En Vivo para atraer la atención de los jóvenes "modernos". Para justificar algunas limitaciones de la campaña, en el mismo documento se menciona la agresiva inversión de Marlboro para dominar el mercado mediante la creación de una imagen "cada vez más joven" ("... Marlboro advertising [...] smoker image and profile are getting younger") ${ }^{21}$. El documento incluye datos sobre la lucha entre las multinacionales y Tabacalera por el mercado joven español.

Aunque las tabaqueras insistieron en que YAS iba dirigido específicamente al adulto joven (de más de 18 años) posiblemente sabían que limitar la propaganda a un grupo de edad determinado es prácticamente imposible, sobre todo en un país como España en que la normativa que prohibía el acceso de menores a cierta publicidad y espectáculos generalmente no era de completa aplicación ${ }^{11,12}$. Pero en cualquier caso, los documentos revelan que la intención real de las tabaqueras no era sólo los adultos. Philip Morris, por ejemplo, invirtió en estudios de mercado con el objetivo de entender el mercado español y elaborar planes de promoción a largo plazo para atraer a nuevos fumadores y adultos jóvenes ("PM EEC BRAND MARKETING SUCCESS [en la Comunidad Económica Europea y España] DEPENDS ON ATTRACTING AND MAINTAINING A HIGH SHARE OF STARTERS AND YOUNG ADULT SMOKERS [...] REGIONWIDE IN-DEPTH RESEARCH WAS CONDUCTED AMONG YOUNG ADULTS [...] : THE MAIN OBJECTIVE WAS TO DEVELOP A CLEAR UNDERSTANDING OF YOUNG ADULT NEEDS, VALUES AND LIFESTYLES AND HOW THEY SEE THE WORLD IN TERMS OF IMAGE ADVERTISING")22. Parte de la estrategia incluia el precio del tabaco, factor que influye más en los menores y jóvenes ("WE NEED TO REGULARLY EVALUATE THE CHANGING BEHAVIOUR, LIFESTYLES AND VALUES OF THESE GROUPS TO DETERMINE THE APPEAL OF OUR BRANDS VS COMPETITORS, MONITOR BRAND IMAGES AND THE ATTRACTION OF PRICE VALUE PROPOSITIONS")22.

En otro informe de 1993, Philip Morris incluía a España entre los 16 mercados más importantes del mundo y mencionaba claramente que el futuro de estos mercados dependía del número de nuevos fumadores ("The long term trend of smoking incidence depends upon the number of starters joining the market and the number of quitters leaving") ${ }^{23}$ ya que la incidencia de fumadores estaba disminuyendo en la mayoría de los países y sobre todo en la Comunidad Económica Europea ("The level of starters is declining in the majority of markets, particularly in the EEC Region") ${ }^{23}$. Ya en los años 90 
Tabla 3. Documentos analizados en este estudio (Legacy Tobacco Documents Library)

\begin{tabular}{|c|c|c|c|c|c|c|c|}
\hline$\#$ & Título & Autor & Fecha & Bates \# & Colección & $\begin{array}{l}\text { Pági- } \\
\text { nas }\end{array}$ & Otra información \\
\hline 1 & The importance of younger adults & RJR & 00000000 & $503473660-503473665$ & Research & 6 & $\begin{array}{l}\text { Special collections FTC Joe } \\
\text { Camel complaint }\end{array}$ \\
\hline 2 & $\begin{array}{l}\text { Winston Americana en vivo advertising } \\
\text { performance - Spain }\end{array}$ & Ruiz AG; RJR Intl & $\begin{array}{l}19860908 \text { (Septem- } \\
\text { ber } 8,1986)\end{array}$ & $515606794 / 6800$ & RJ Reynolds & 7 & Graphics; letter \\
\hline 3 & $\begin{array}{l}\text { Brand marketing } 3 \text { year plan strategic } \\
\text { review }\end{array}$ & PM-EEC & $\begin{array}{l}19920900 \text { (Septem- } \\
\text { ber, 1992) }\end{array}$ & $2501025371 / 5430$ & Philip Morris & 60 & $\begin{array}{l}\text { Brpl, brand plan ; char, chart, } \\
\text { graph, table, maps }\end{array}$ \\
\hline 4 & $\begin{array}{l}\text { The Marlboro monitor } 880000 \text { - } \\
920000 \text { executive summary }\end{array}$ & $\begin{array}{l}\text { PMI, Philip Morris } \\
\text { International }\end{array}$ & $\begin{array}{l}19930700 \text { (July, } \\
\text { 1993) }\end{array}$ & $2500141457 / 1494$ & Philip Morris & 38 & $\begin{array}{l}\text { Spch, speech, presentation; } \\
\text { rept, report, other }\end{array}$ \\
\hline 5 & Winston advertising campaign & Lopez I & $\begin{array}{l}\text { 19920600 (June, } \\
\text { 1992) }\end{array}$ & $525912395 / 2399$ & RJ Reynolds & 5 & Report \\
\hline 6 & PM-EEC marketing review & PM-EEC & $\begin{array}{l}19920500 \text { (May, } \\
1992)\end{array}$ & $2501058841 / 8951$ & Philip Morris & 111 & Brre, brand review \\
\hline 7 & Project Itn outline & PM-EEC & $\begin{array}{l}\text { 19930600 (June, } \\
\text { 1993) }\end{array}$ & $2501151345 / 1358$ & Philip Morris & 14 & $\begin{array}{l}\text { Mrrt, market research } \\
\text { report; char, chart, graph, } \\
\text { table, maps; outl, outline; } \\
\text { spch, speech, presentation }\end{array}$ \\
\hline 8 & Spain mainland Marlboro medium & No indicado & $\begin{array}{l}19951100 \text { (Novem- } \\
\text { ber, 1995) }\end{array}$ & 2046650818/0819 & Philip Morris & 2 & Brre, brand review \\
\hline 9 & Bright cigarette blend & Lowe, RD & $\begin{array}{l}19830210 \text { (February } \\
10,1983)\end{array}$ & 105153966-105153968 & $\begin{array}{l}\text { British American } \\
\text { Tobacco }\end{array}$ & 3 & Letter \\
\hline 10 & Information about lights in Spain & $\begin{array}{l}\text { Losada P (BAT España } \\
\text { SA) }\end{array}$ & $\begin{array}{l}19940923 \text { (Septem- } \\
\text { ber 23, 1994) }\end{array}$ & $500236841-500236843$ & $\begin{array}{l}\text { British American } \\
\text { Tobacco }\end{array}$ & 3 & Note \\
\hline 11 & $\begin{array}{l}\text { The findings from a qualitative study } \\
\text { in the Sweden, Spain and Italy that } \\
\text { investigated attitudes to good business } \\
\text { practice }\end{array}$ & Philip Morris & $\begin{array}{l}\text { 19980705 (July } 5, \\
\text { 1998) }\end{array}$ & 2074189094/9163 & Philip Morris & 70 & $\begin{array}{l}\text { Spch, speech, presentation; } \\
\text { manu, manual, handbook, } \\
\text { catalogue; rept, report, other }\end{array}$ \\
\hline 12 & Youth smoking & Lindheim J & $\begin{array}{l}19960303 \text { (March } \\
3,1996)\end{array}$ & $2501209627 / 9628$ & Philip Morris & 2 & Memo, memorandum \\
\hline 13 & $\begin{array}{l}\text { Following are highlights of February } \\
\text { external relations activities and issues } \\
\text { worldwide }\end{array}$ & Griscom TC & $\begin{array}{l}19970305 \text { (March } \\
5,1997)\end{array}$ & $531455274 / 5283$ & RJ Reynolds & 10 & Email; letter \\
\hline 14 & Monthly E.R. report no 2 - Spain. & Barron A; RJR Intl & $\begin{array}{l}19970226 \text { (February } \\
26,1997)\end{array}$ & $528373595 / 3598$ & RJ Reynolds & 4 & Email; letter \\
\hline 15 & $\begin{array}{l}\text { RJRI. International tobacco's perfor- } \\
\text { mance for the quarter and year was } \\
\text { impacted by the strength of }\end{array}$ & & $\begin{array}{l}19980122 \text { (January } \\
22,1998)\end{array}$ & $528745467 / 5468$ & RJ Reynolds & 2 & Email; report \\
\hline 16 & $\begin{array}{l}\text { Social issues service } 990000 \text { company } \\
\text { report - e RJR Nabisco holdings corp. } \\
\text { Tobacco production and marketing }\end{array}$ & $\begin{array}{l}\text { Investor Responsibil- } \\
\text { ity Research Center }\end{array}$ & 19990000 (1999) & 2077407304/7327 & Philip Morris & 24 & $\begin{array}{l}\text { Rept, report, other; char, } \\
\text { chart, graph, table, maps }\end{array}$ \\
\hline 17 & $\begin{array}{l}\text { Following are highlights of august } \\
\text { external relations activities and issues } \\
\text { worldwide: United States. }\end{array}$ & Griscom TC & $\begin{array}{l}19970903 \text { (Septem- } \\
\text { ber } 3,1997)\end{array}$ & $517115934 / 5942$ & RJ Reynolds & 9 & Letter \\
\hline
\end{tabular}

existía sobrada evidencia de que la mayoría de los fumadores comienzan antes de los 18 años $^{24}$, y es por ello que la estrategia que Philip Morris propuso fue rejuvenecer las campañas de promoción para hacerlas atractivas no sólo a los adultos jóvenes, sino también a los nuevos fumadores ("Marlboro communications need to be refreshed to regain greater appeal and relevance to starters and the young adult smoker target audience") ${ }^{23}$. Igualmente, RJ Reynolds insistió en la necesi- dad de "rejuvenecer" las campañas de promoción de Winston en España ("The new communication should also be impactful and provoke interest and add youth and life enjoyment to the brand") ${ }^{25}$.

Otro aspecto interesante relacionado con el diseño son los cigarrillos bajos en nicotina y alquitrán, que las tabaqueras comenzaron a introducir en Estados Unidos en los años 60 como respuesta a las llamadas de las autoridades de salud 
pública y con el mensaje de que suponían una alternativa más segura. Estudios posteriores ${ }^{26-29}$ han mostrado que las tabaqueras intencionadamente confundieron al fumador, ya que sus propios experimentos habían determinado que los niveles de nicotina y alquitrán estimados con máquinas y en laboratorio no reflejan la realidad ya que los fumadores modifican su hábito para mantener en su organismo la misma cantidad de nicotina a la que están acostumbrados. Mediante propaganda las tabaqueras crearon una imagen falsa de estos cigarrillos, y las consecuencias de salud pública fueron nefastas ya que muchos fumadores decidieron continuar en lugar de abandonar el tabaquismo ${ }^{26-29}$.

Lo que no se ha discutido claramente en la literatura es que las tabaqueras también utilizan los cigarrillos bajos en nicotina y alquitrán para atraer a fumadores jóvenes. Los documentos muestran que ésa fue la estrategia utilizada con éxito en España. En dos informes de 1992 y 1993 sobre Europa, Philip Morris explicaba cómo en los mercados clave, entre los que se incluía España, los nuevos fumadores y adultos jóvenes rechazaban el sabor fuerte del tabaco natural y preferian las versiones bajas en nicotina y alquitrán (LTN en sus siglas en inglés) ("More starters begin with $\left.L T N{ }^{\prime} \mathrm{s}^{\prime}\right)^{30}$, y la necesidad de crear una imagen de mercado adecuada ("In key markets more starters and Y.A.S. are smoking LTN's. We must have products with brand images that appeal to this target market") ${ }^{31}$. Estos documentos muestran además que Philip Morris distinguía claramente entre nuevos fumadores, generalmente menores de edad iniciándose al tabaquismo, y adultos jóvenes y que sus campañas de promoción iban destinadas a ambos grupos. La estrategia no tardó en dar fruto y ya en 1995 la multinacional explicaba en un informe sobre España cómo sus productos bajos en nicotina y alquitrán estaban atrayendo un mayor número de nuevos fumadores ("LTN is attracting an increasing number of starters") ${ }^{32}$. Igualmente importante es reiterar que los ingredientes de los cigarrillos, sobre todo los productos que se añaden para disfrazar los efecto irritantes del tabaco en las mucosas y darle un sabor menos desagradable, influyen en cómo se reacciona al primer cigarrillo y por tanto en la incidencia de fumadores jóvenes ${ }^{33}$. Las grandes multinacionales elaboraron fórmulas de sabores conteniendo azúcar, regaliz, miel y otros ingredientes que en colaboración con Tabacalera se utilizaron en España $^{34}$.

La falsa imagen de los cigarillos "light" también influyó en las mujeres españolas, y las tabaqueras no dejaron pasar la oportunidad. En un documento de 1994, British American Tobacco España explicaba que las españolas tenían una actitud más favorable [que los hombres] hacia los cigarrillos bajos en alquitrán y los preferían en parte porque creían que reducían los efectos nocivos para la salud como tos, irritación de garganta, dificultad respiratoria y hacer ejercicio ("They reduce harmful effects: coughing, throat harshness, difficulty in breathing, playing sports") y representaban un símbolo de adhesión al progreso y la vida moderna ("women seem to adopt Low-Tar delivery cigarettes with a more favorable attitude, which also represents a symbolic adhesion to progress and modernity") ${ }^{35}$.
Otro aspecto importante para las tabaqueras es la imagen social. Como es sabido, las tabaqueras han empleado siempre una estrategia combinada en la que utilizan la buena imagen social de la compañía para promocionar el tabaquismo entre los jóvenes. La imagen positiva de la que la compañía Philip Morris disfrutó en España durante décadas ("In both Spain and Italy, Philip Morris has a very positive image: a highly successful company; very good brands; a good employer; impeccable business practices..." ${ }^{36}$ no fue una casualidad, sino el resultado de un cuidado plan de acción basado en estudios de opinión. Cuando a finales de los años 80 Philip Morris dedujo que la sociedad española empezaba a pedirle medidas concretas para proteger del tabaco a los jóvenes, enseguida elaboró estrategias de distracción y engaño que mantuvieran su buena imagen sin afectar la incidencia de nuevos fumadores ("The other major problem is Youth. People's belief that a cigarette company needs to persuade a new generation to take up smoking is the toughest obstacle to them seeing Philip Morris as a good citizen. For the company to be seen to be behaving in a responsible way on this issue will help [...]. It may also help if people can begin to see Philip Morris as a large multi-national, whose cigarette business is but a part of a wide portfolio of other products. This would make the company seem less dependent on seducing a new generation to smoking") ${ }^{36}$.

Más conocidos son los programas destinados a impedir a los menores de edad la compra de tabaco, programas que no afectan la prevalencia entre los jóvenes y sí benefician la imagen de las compañias ${ }^{37}$. En España, las tabaqueras financiaron programas para jóvenes con la única intención de mejorar su imagen social e interferir con las políticas de control ("I was surprised how few of them put much emphasis on "working with others to develop communications programs aimed at youth to discourage them from smoking". This is [...] sort of hinted at in the Belgian, Italian, and Spanish programs. This area is to me the untapped possibility on this issue since it should be an approach that a) "everyone can agree on" including the trade, the government, the industry, b) makes a visible and tangible effort which the public could feel and touch; and c) is in no way incompatible with all of our positions on "why kids smoke" and how to effectively deal with the problem. By focusing on this approach, one can draw attention to the real reasons why kids want to smoke, thereby robbing the other side of the ability to focus this issue on the wrong solutions, e.g., fiscality") ${ }^{38}$. Un comunicado interno de RJ Reynolds de 1997 refleja perfectamente este doble juego que las multinacionales emplearon en España, por un lado, y en colaboración con Tabacalera, promocionando programas que supuestamente protegían a los menores (" $R J R$ Canary Islands has initiated discussions with BAT and PMI to implement a Minors Program in line with the one rolled out in Spain's mainland. Tabacalera has been contacted in Madrid and also agreed with the initiative") ${ }^{39}$ y por otro intentando bloquear las restricciones a la propaganda ("In an effort to persuade the Spanish Government to change its position regarding tobacco advertising, the Spanish Association of Advertising Agencies has published a book titled "Tobacco and Advertising," ${ }^{39}$. 
Otra estrategia publicitaria cuyo efecto en los jóvenes no se ha investigado suficientemente en nuestro país es la campaña Camel Tips, con la que RJ Reynolds sustituyó al tristemente famoso Joe Camel. La campaña se lanzó en Europa en 1996 y en España en 1997 ("Coinciding with the launch of the Camel Tips campaign, a P.R. plan has been implemented in order to build campaign awareness, to maximize magazines investment synergies, and to ensure an adequate consumer campaign understanding through media coverage") ${ }^{40}$, y en 1998 la propia compañia informaba de su repercusión positiva en las ventas ("International tobacco's performance for the quarter and year was impacted by [...], temporary price disruption in France and Spain and difficulties with the Russian export pipeline. Volume trends began to improve towards the end of the year with new and recent brand programs- [...], Camel Tips [...] -continuing to do well" ${ }^{41}$. Según RJ Reynolds, Camel Tips pretendía atraer a los adultos jóvenes mediante el humor aunque su personaje central era un muñeco de peluche no muy diferente de Joe Camel y obviamente atractivo a niños y menores. No sólo RJ Reynolds tuvo que defender la campaña ante algunos tribunales europeos, sino que sus propios accionistas ${ }^{42}$ y otras tabaqueras cuestionaron la legalidad de la campaña ("BAT has accused RJR of breaching legal provisions (i .e., a ban on health benefit claims and ban on ads attractive to minors) with two Camel Tips ads. RJR is defending its ads by all legal means") ${ }^{43}$.

\section{Limitaciones}

Hay que indicar que la investigación de los documentos internos de las tabaqueras tiene como principal limitación la enorme cantidad de documentos archivados en las distintas bases de datos. El número de documentos relacionados con España es enorme, una reciente publicación ha calculado más de $85.000^{44}$. A pesar de los avanzados mecanismos de búsqueda electrónica, las pantallas de resultados incluyen por lo general múltiples duplicaciones del mismo documento lo que dificulta aún más la selección y revisión. Los resultados de este estudio podrían por tanto estar en cierta manera sesgados si no se hubieran identificado acertadamente documentos clave relacionados con el tema del estudio. Creemos, sin embargo, que la metodología utilizada reduce este riesgo ya que hemos revisado más de 1.000 documentos y los que se analizaron fueron suficientes para apoyar de forma coherente las conclusiones del estudio. Este análisis se basa exclusivamente en los documentos procedentes de los archivos de las grandes tabaqueras entre los que existen algunos relacionados con compañías españolas como Tabacalera S.A. Desafortunadamente no se dispone de una base de datos exclusivamente de documentos relacionados con España.

\section{CONCLUSIONES}

Ya en los años 60 las grandes compañías tabaqueras decidieron que para mantener su nivel de negocio tenían que seguir atrayendo a nuevos fumadores. Miles de documentos muestran la intensa actividad que durante las décadas de los 80 y 90 mantuvieron las compañías a nivel internacional en torno a los "adultos jóvenes", y es muy posible que estas campañas influyeran también en otros grupos de edad. En Estados Unidos por ejemplo, un reciente estudio longitudinal sobre uso del tabaco entre adolescentes concluyó que entre 2002 y 2008 la incidencia de tabaquismo permaneció constante y que los esfuerzos para prevenir que los adolescentes empiecen a fumar necesitan incrementarse ${ }^{45}$.

En España existe la percepción general de que las actividades de las grandes tabaqueras no nos afectaron ni afectan. Los documentos muestran que las multinacionales del tabaco aplicaron las mismas tácticas en todos los países y que en España no actuaron independientemente sino que colaboraron entre ellas y con Tabacalera y otras compañias nacionales. Los documentos identificados y analizados en este estudio corroboran que así actuaron durante las décadas de los 80 y 90 para atraer a fumadores jóvenes, y las consecuencias de ese plan conjunto quizás haya influido en las tasas actuales de tabaquismo en España.

Las tabaqueras han actuado y actúan de forma coordinada para captar fumadores jóvenes e impedir las políticas de control con el propósito de mantener su lucrativo negocio. Dada la intensa actividad que las tabaqueras llevaron a cabo en torno a los jóvenes hasta finales de los 90, la salud pública española debería prepararse para afrontar una nueva generación de fumadores e intensificar ya sus programas de educación y abandono del tabaquismo.

Los resultados de este estudio también muestran que la investigación de los documentos de las tabaqueras es una estrategia que puede contribuir a la lucha contra el tabaquismo en España. En definitiva, los documentos que aquí se presentan ayudan a desvelar las prácticas poco éticas de las compañías para promocionar sus productos entre los adolescentes e interferir con las políticas de control, como la regulación de espacios sin humo o las limitaciones al patrocinio, promoción y publicidad.

\section{Agradecimientos}

Parte de la información aquí incluida está basada en el informe La industria tabaquera y la promoción del tabaquismo entre los menores ${ }^{16}$, que el primer autor elaboró en 2006 para la Junta de Andalucia en apoyo de su demanda contra las compañías tabaqueras reclamando el coste incurrido por el sistema sanitario andaluz como consecuencia de las enfermedades derivadas del tabaquismo. La demanda está todavía pendiente. La Junta de Andalucía no ha influido en ningún sentido sobre los autores acerca de los resultados presentados en aquel informe ni en el presente trabajo. Los autores no declaran conflictos de intereses.

Queremos expresar nuestro agradecimiento a Francisco Javier García León y a la Consejería de Salud de la Junta de Andalucía por su interés en esta línea de investigación. 


\section{Referencias}

1. Peto $R_{1}$ Lopez AD. The future worldwide health effects of current smoking patterns. En: Boyle P, Gray N, Henningfield J, Seffrin J, Zatonski W, editors. Tobacco and public health: science and policy. Oxford: Oxford University Press; 2004. p. 281-6.

2. Delegación del Gobierno para el PNSD. Informe de la Encuesta Estatal sobre uso de Drogas en Estudiantes de Enseñanzas Secundarias (ESTUDES) 2008. Ministerio de Sanidad y Política Social; 2009. Disponible en: http//www.pnsd.msc.es/ Categoria2/observa/pdf/Estudes2008_Web.pdf; consultado el 1 de junio de 2011.

3. Soto Mas F, Villalbi JR, Balcázar H, Valderrama Alberola J. La iniciación al tabaquismo: aportaciones de la epidemiología, el laboratorio y las ciencias del comportamiento. An Esp Pediatr 2002; 57: 327-33.

4. Nebot $M_{1}$ Tomás $Z$, Ariza $C$, Valmayor $S$, López MJ, Juárez 0 . Factores asociados con el inicio del tabaquismo: seguimiento a los 3 años de una cohorte de escolares. Arch Bronconeumol 2004; 40: 495-501.

5. Calafat A, Fernández Gómez C, Juan M, Becoña E. Vida recreativa nocturna de los jóvenes españoles como factor de riesgo frente a otros más tradicionales. Adicciones 2007; 19: 125-32.

6. Granero L, Villalbi JR, Gallego R. ¿Quién se opone a la prevención? Un mapa de los actores pro tabaco en España. Gac Sanit 2004; 18: 374-9.

7. Pollay RW, Siddarth S, Siegel M, Haddix A, Merritt RK, Giovino $G A$, et al. The last straw? Cigarette advertising and realized market shares among youths and adults, 1979-1993. Journal of Marketing 1996; 60: 1-16.

8. Salvador-Llivina T. Condicionantes del consumo de tabaco en España. Adicciones 2004; 16 (Supl 2): 25-58.

9. Montes J, Álvarez ML. Tabaco y medios de comunicación escritos en España: una atracción fatal. Gac Sanit 2006; 20: 59-62.

10. de Castro FJ, Hernández-Mezquita MA, Rivas JP, González MT, Puerto MJ, Adeva MT. Publicidad del tabaco en los medios de comunicación escritos. Prev Tab 2001; 3: 138-42.

11. Sarría-Santamera A, Cortés-Blanco $M$, Elder J. Análisis de la campaña publicitaria de la marca de tabaco Fortuna durante el bienio 1999-2000. Rev Esp Salud Pública 2001; 75: 107-14.

12. Mosella MC, Villalbi JR, Hayes A, Nebot M. Sobre jóvenes, revistas, publicidad y educación sanitaria. Gac Sanit 1999; 13: 166-8.

13. Ayesta FJ, Sáez E. Tabaquismo: una adicción que nos concierne. Trastornos Adictivos 2004; 6: 71-7.

14. Tamborero Cao G. Magnitud de la promoción publicitaria de tabaco y bebidas alcohólicas en una muestra de los semanarios españoles. Rev San Hig Púb 1991; 65: 137-46.

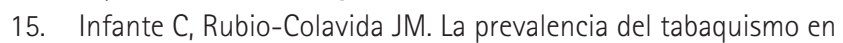
España. Adicciones 2004; 16 (Supl 2): 59-73.

16. Soto Mas F, García León FJ. La industria tabaquera y la promoción del tabaquismo entre los menores y jóvenes: una revisión internacional. Gac Sanit 2009; 23: 448-57.

17. Soto Mas F. La industria tabaquera y la promoción del tabaquismo entre los menores. Informe Técnico. Sevilla: Junta de Andalucia (España); 2006.

18. Soto Mas F, Villalbi J, Granero L, Jacobson H, Balcázar H. Los documentos internos de la industria tabaquera y la prevención del tabaquismo en España. Gac Sanit 2003; 17 (Supl 3): 9-14.
19. Campaign for Tobacco-Free Kids. Big tobacco's Guinea pigs: how an unregulated industry experiments on America's kids and consumers. American Heart Association, American Lung Association, American Cancer Society. Disponible en: www. tobaccofreekids.org/reports/products; consultado el 1 de junio de 2011.

20. RJ Reynolds. The importance of younger adults. Fecha no indicada. RJ Reynolds Tobacco Company. Bates \# 503473660503473665. Disponible en: http://legacy.library.ucsf.edu; consultado el 1 de junio de 2011.

21. Ruiz AG. Winston Americana en vivo advertising performanceSpain. Fecha 8 septiembre, 1986. RJ Reynolds Tobacco Company. Bates \# 515606794/6800. Disponible en: http://legacy.library. ucsf.edu; consultado el 1 de junio de 2011.

22. Philip Morris-EEC. Brand marketing 3 year plan strategic review. Fecha septiembre, 1992. Philip Morris Companies, Inc. Bates \# 2501025371/5430. Disponible en: http://legacy.library.ucsf.edu; consultado el 1 de junio de 2011.

23. Philip Morris International. The Marlboro monitor 880000 920000 executive summary. Fecha julio, 1993. Phillip Morris Companies, Inc. Bates \# 2500141457/1494. Disponible en: http://legacy.library.ucsf.edu; consultado el 1 de junio de 2011.

24. U.S. Department of Health and Human Services. Preventing tobacco use among young people: a report of the Surgeon General. Atlanta, Georgia: DHHS, Public Health Service, Centers for Disease Control and Prevention, National Center for Chronic Disease Prevention and Health Promotion, Office on Smoking and Health; 1994.

25. Lopez I. Winston advertising campaign. Fecha: junio, 1992. RJ Reynolds Tobacco Company. Bates \# 525912395/2399. Disponible en: http://legacy.library.ucsf.edu; consultado el 1 de junio de 2011.

26. National Cancer Institute. Smoking and Tobacco Control Monograph 13: Risks associated with smoking cigarettes with low machine-measured yields of tar and nicotine. Bethesda, MD: NCl; 2001.

27. U.S. Department of Health and Human Services. The health consequences of smoking: a report of the Surgeon General. [Atlanta, Ga.]: DHHS, Centers for Disease Control and Prevention, National Center for Chronic Disease Prevention and Health Promotion, Office on Smoking and Health; 2004.

28. Rigotti NA, Tindle HA. The fallacy of 'light' cigarettes. BMJ USA 2004; 4:E278-79.

29. Pollay RW, Dewhirst T. The dark side of marketing seemingly "Light" cigarettes: successful images and failed fact. Tob Control 2002; 11 (Suppl I): i18-i31.

30. Philip Morris-EEC. PM-EEC marketing review. Fecha mayo, 1992. Philip Morris Companies, Inc. Bates \# 2501058841/8951. Disponible en: http://legacy.library.ucsf.edu; consultado el 1 de junio de 2011.

31. Philip Morris-EEC. Project Itn outline. Fecha junio, 1993. Philip Morris Companies, Inc. Bates \# 2501151345/1358. Disponible en: http://legacy.library.ucsf.edu; consultado el 1 de junio de 2011.

32. Philip Morris. Spain mainland Marlboro medium. Fecha noviembre, 1995. Philip Morris Companies, Inc. Bates \# 2046650818/0819. Disponible en: http://legacy.library.ucsf.edu; consultado el 1 de junio de 2011. 
33. Dachille K. Pick your poison: responses to the marketing and sale of flavored tobacco products. Tobacco Control Legal Consortium; 2009.

34. Lowe, RD. Bright cigarette blend. Fecha 10 febrero, 1983. British American Tobacco Company. Bates \# 105153966-105153968. Disponible en: http://legacy.library.ucsf.edu; consultado el 1 de junio de 2011.

35. Losada P. Information about lights in Spain. Fecha 23 septiembre 1994. British American Tobacco Company. Bates \# 500236841-500236843. Disponible en: http://legacy.library. ucsf.edu; consultado el 1 de junio de 2011.

36. Philip Morris. The findings from a qualitative study in the Sweden, Spain and Italy that investigated attitudes to good business practice. Fecha julio 5, 1998. Philip Morris Companies, Inc. Bates \# 2074189094/9163. Disponible en: http://legacy. library.ucsf.edu; consultado el 1 de junio de 2011.

37. Ling PM, Landman A, Glantz SA. It is time to abandon youth access tobacco programmes (Editorial). Tob Control 2002; 11: 3-6 doi:10.1136/tc.11.1.3.

38. Lindheim J. Youth smoking. Fecha 3 marzo, 1996. Philip Morris Companies, Inc. Bates \# 2501209627/9628. Disponible en: http://legacy.library.ucsf.edu; consultado el 1 de junio de 2011.

39. Griscom TC. Following are highlights of February external relations activities and issues worldwide. Fecha marzo 5, 1997. RJ Reynolds Tobacco Company. Bates \# 531455274/5283. Disponible en: http://legacy.library.ucsf.edu; consultado el 1 de junio de 2011.

40. Barron A. Monthly E.R. Report no 2 - Spain. Fecha febrero 26, 1997. RJ Reynolds International. Bates \# 528745467/5468. Disponible en: http://legacy.library.ucsf.edu; consultado el 1 de junio de 2011.

41. Bryan Brown A. RJRI. [International tobacco's performance...] Fecha enero 22, 1998. RJ Reynolds International. Bates \# 528745467/5468. Disponible en: http://legacy.library.ucsf.edu; consultado el 1 de junio de 2011.

42. Investor Responsibility Research Center. Social issues service 990000 company report - e RJR Nabisco holdings corp. Tobacco production and marketing. Fecha 1999. Philip Morris Companies, Inc. Bates \# 2077407304/7327. Disponible en: http://legacy.library.ucsf.edu; consultado el 1 de junio de 2011.

43. Griscom TC. Following are highlights of august external relations activities and issues worldwide: United States. Fecha septiembre 3, 1997. RJ Reynolds International. Bates \# 517115934/5942. Disponible en: http://legacy.library.ucsf.edu; consultado el 1 de junio de 2011.

44. Seffrin JR, Baldini P. The tobacco atlas online. World Lung Foundation, American Cancer Society; (n.d.). Disponible en: http://www.tobaccoatlas.org/default.html; consultado el 1 de junio de 2011.

45. Substance Abuse and Mental Health Services Administration. The NSDUH Report: trends in tobacco use among adolescents. 2002 to 2008. Office of Applied Studies. Rockville, MD; 2009. 